\title{
Efecto del ácido alfa lipoico y la pirfenidona en la modulación antioxidante celular contra el daño oxidativo
}

\author{
JOSÉ MACIAS-BARRAGAN ${ }^{1, \mathrm{a}}$, ALESSANDRA CALIGIURI ${ }^{2, \mathrm{a}}$, \\ JESÚS GARCÍA-BANUELOS ${ }^{3, a}$, MAURIZIO PAROLA ${ }^{4, a}$, \\ MASSIMO PINZANI MD,a, JUAN ARMENDARIZ-BORUNDA ${ }^{3, a}$
}

\section{Effects of alpha lipoic acid and pirfenidone on liver cells antioxidant modulation against oxidative damage}

Background: Liver fibrogenic processes are related to cellular redox state. Glutathione (GSH) is the major cellular antioxidant. GSH induced activation could be related to antifibrogenic effects. Aim: To explore the association between the antifibrogenic effect and pro-antioxidant mechanisms of alpha-lipoic acid (ALA) and pirfenidone (PFD). Material and Methods: HepG2 cells and primary HSC cultures were exposed to menadione $0.1 \mu M(M E N)$ as oxidative stress inducer and treated to ALA (5 mM) or PFD (10 $\mu \mathrm{M}, 100 \mu \mathrm{My} 1000 \mu \mathrm{M})$. Results: In HSC, PFD decreased cell proliferation and the expression of COL1A1, TGF- $\beta 1$, TIMP1, IL6, TNF $\alpha$ and MCP1 induced by MEN. Furthermore it was confirmed that ALA and PFD activate diverse antioxidants mediators, however MEN decreases this response. Then, MEN, ALA and PFD induce an antioxidant response, the first one as a response to injury and the latter two as pro-antioxidant inducers. Therefore, when cells are exposed to oxidative stress, endogenous systems activate a battery of mediators that increase the antioxidant potential. When these cells are treated with ALA and PFD, de novo formation of protective genes decreases since previous elicited protection induced in response to injury, enhance ALA and PFD effects. Conclusion: Regardless of the route of action, ALA and PFD induce the biosynthesis of antioxidants mediators which is associated with modulation of fibrogenic processes.

(Rev Med Chile 2014; 142: 1553-1564)

Key words: Fibrosis; Glutathione Hepatic stellate cells; Pirfenidone; Thioctic acid.

$\mathrm{L}$ os sistemas antioxidantes modulan ciertos procesos inflamatorios y fibrogénicos durante el establecimiento de hepatopatías, lo que podría ser utilizado en el tratamiento de la fibrosis. Sin embargo, existe una gran cantidad de protocolos clínicos en los que la inactivación

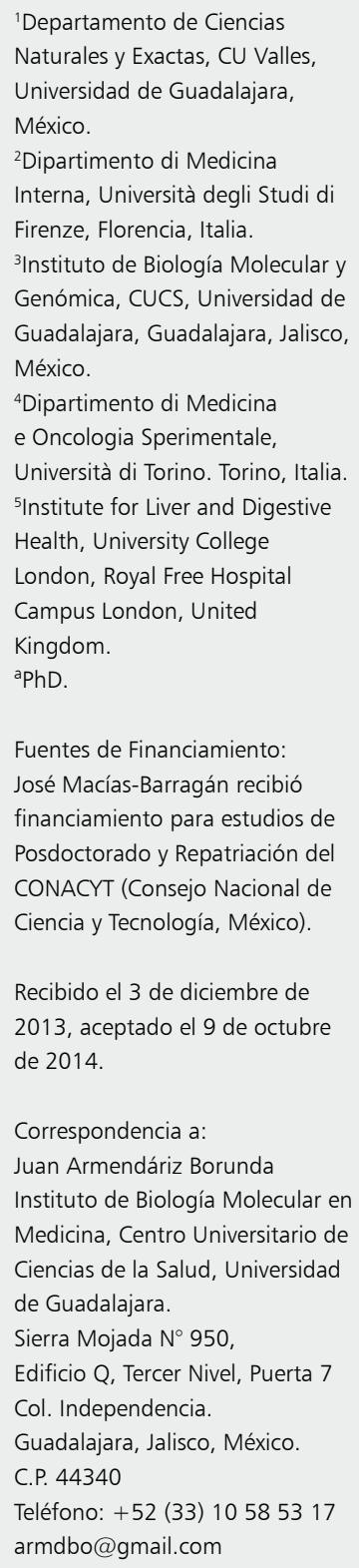

Fuentes de Financiamiento: José Macías-Barragán recibió financiamiento para estudios de Posdoctorado y Repatriación del CONACYT (Consejo Nacional de Ciencia y Tecnología, México).

Recibido el 3 de diciembre de 2013, aceptado el 9 de octubre de 2014.

\section{Correspondencia a:} Juan Armendáriz Borunda Instituto de Biología Molecular en Medicina, Centro Universitario de Ciencias de la Salud, Universidad de Guadalajara.

Sierra Mojada $\mathrm{N}^{\circ} 950$,

Edificio Q, Tercer Nivel, Puerta 7

Col. Independencia.

Guadalajara, Jalisco, México. C.P. 44340

Teléfono: +52 (33) 10585317

armdbo@gmail.com

de las especies reactivas del oxígeno (ROS, del inglés reactive oxygen species) por moléculas antioxidantes es poco efectiva, por lo que es necesario desarrollar estrategias para la disminución de ROS durante la fibrogénesis.

Las células estelares hepáticas (HSC, del in- 
glés hepatic stellate cells) son el tipo celular más relevante durante la fibrogénesis ${ }^{1}$. Durante lesiones hepáticas las HSC se activan y proliferan, generando matriz extracelular (MEC) y citocinas profibrogénicas, las cuales son moduladas por reacciones dependientes de ROS, ya que el aumento del estrés oxidativo es una de las principales vías para la activación de las HSC. El factor de transcripción nuclear eritroide-2 (NF-E2) (Nrf2, del inglés: nuclear factor erythroid 2-related factor) regula la transcripción de genes antioxidantes ${ }^{2-4}$. Nrf2 se encuentra constitutivamente modulado por Keap1, a través de mecanismos que impiden o reducen su interacción, proceso dependiente del estado redox del medio o la intervención de compuestos "pro-antioxidantes". En algunas células, la transcripción génica inducida por Nrf2 depende de los mecanismos que interfieren con la interacción intranuclear entre Nrf2-Keap1 ${ }^{5-7}$ para permitir la unión de Nrf2 a las regiones reguladoras de los genes diana ${ }^{8}$. La activación de Nrf2 también puede ser mediada por la inducción de la vía de la cinasa c-Jun N-terminal (JNK) ${ }^{9-14}$.

El metabolismo de xenobióticos a menudo se divide en las fases de modificación (I), conjugación (II) y excreción (III). Estas reacciones actúan en concierto para inactivar xenobióticos y excretarlos. El factor transcripcional Nrf2 promueve la expresión de mediadores de la fase II, donde los subproductos de la fase I son inactivados mediante conjugación con ligandos como el glutatión $(\mathrm{GSH})$, principal antioxidante celular endógeno sintetizado a partir de L-cisteína, ácido L-glutámico y glicina. La modulación recíproca entre los mediadores de la fase I, II y III pueden compartir mecanismos similares en diferentes tipos de células ${ }^{15,16}$.

El grupo sulfhidrilo ( $\mathrm{SH}$ ) de la cisteína, sirve como donador de átomos de hidrógeno y es responsable de la actividad biológica. El GSH se sintetiza por la acción de las enzimas gamma-glutamil-cisteína sintetasa $(\gamma$-GCS) y glutatión sintetasa (GS). La $\gamma$-GCS es una enzima heterodimérica compuesta por una subunidad catalítica (GCLC) codificada por el gen GCLC, y una subunidad moduladora (GCLM) codificada por el gen GCLM. La transcripción de GCLC y GCLM es inducida por Nrf2 y se incrementa en procesos que cursan con estrés oxidativo y daño celular ${ }^{17}$. Sin embargo, no solamente los genes antioxidantes son transcritos durante procesos de daño, la activación de factores de transcripción como el NF-kB se relaciona con la evolución y la perpetuación del proceso inflamatorio ${ }^{18}$, promoviendo la activación de cinasas (ERK, JNK, AMPK y Akt) que favorecen la producción de mediadores fibrogénicos, entre los más importantes, el factor de crecimiento derivado de plaquetas (PDGF) y el factor de crecimiento transformante- $\beta 1$ (TGF$\beta 1)^{19-22}$. Por lo tanto, variaciones en la regulación de genes antioxidantes contribuyen directamente en la activación de las HSC ${ }^{23,24}$.

La pirfenidona (PFD, 5-metil-1-fenil-2-(1H)piridona) es un fármaco que ha demostrado un eficaz efecto antifibrótico ${ }^{25}$ y anti-inflamatorio en varios modelos animales, in vitro y en ensayos clínicos de enfermedades fibrogénicas ${ }^{26}$. Se ha observado que la PFD posee la capacidad de neutralizar directamente ciertos radicales libres, como el radical superóxido $\left(\mathrm{O}_{{ }^{-}}^{-}\right)$, lo que puede contribuir a las actividades antifibróticas de esta molécula ${ }^{27}$. Además ha demostrado un incremento en la capacidad antioxidante celular, pero el mecanismo molecular exacto es desconocido ${ }^{28}$. Por lo que su acción protectora es inducida por varias vías. Con base en esto, la idea central que surge es que, además del potencial antifibrogénico, induce la formación de novo de proteínas anti-oxidantes. Se ha observado que ciertos compuestos, como el ácido lipoico (ALA), inducen esta respuesta ${ }^{29}$. Por lo tanto, este estudio tiene como objetivo definir el mecanismo molecular por el cual la PFD modula la vía de señalización antioxidante en células hepáticas durante un proceso de estrés oxidativo inducido por la menadiona (MEN).

\section{Materiales y Métodos}

\section{Reactivos}

El anticuerpo policlonal Nrf2 es de Santa Cruz Biotecnology (Santa Bárbara, CA) y el anticuerpo monoclonal para fibrilarina de Sigma (St. Louis, MO). El PDGF humano recombinante de Peprotech (Rock Hill, NJ). El medio de cultivo Dulbecco's Modified Eagle Medium (DMEM) e Iscove's Modified Eagle's Medium (IMEM), glutamax, aminoácidos no esenciales y tripsina fueron obtenidos de Gibco BRL Life Technologies (Grand Island, NY) y el suero bovino fetal (FBS) de Hyclone Laboratories (Logan, RU). Si no se especifica, todos los demás reactivos son de grado analítico de Sigma (St. Louis, MO). 


\section{Diseño experimental}

Para evaluar el efecto de la PFD a diferentes dosis $(10 \mu \mathrm{M}, 100 \mu \mathrm{M}$ y $1.000 \mu \mathrm{M})$ o $\operatorname{MEN}(0,1$ $\mu \mathrm{M})$, se analizó la cinética de la actividad metabólica mitocondrial (ensayo de MTT) de las HSC a las 12, 24 y $48 \mathrm{~h}$ posteriores a la exposición. En otro diseño experimental se analizó el efecto de PFD $(100 \mu \mathrm{M})$ y ALA $(5 \mathrm{mM})$ en la actividad metabólica mitocondrial inducida por PDGF (10 $\mathrm{ng} / \mathrm{ml})$ o MEN $(0,1 \mu \mathrm{M})$ a las $8 \mathrm{~h}$. Para evaluar la expresión de novo (qRT-PCR) de genes pertenecientes a las categorías antioxidante, inflamatoria y fibrogénica, primero las HSC fueron expuestas a PFD $(100 \mu \mathrm{M})$ o ALA (5 mM) por $2,4,8,12$ y $24 \mathrm{~h}$. Posteriormente se analizó el efecto de PFD $(10 \mu \mathrm{M}, 100 \mu \mathrm{M}$ y $1.000 \mu \mathrm{M})$ sobre la modulación de la expresión génica de estas categorías en HSC expuestas a MEN $(0,1 \mu \mathrm{M})$ por $8 \mathrm{~h}$. Los niveles de GSSG, GSH y GSH total en las HSC fueron cuantificados $24 \mathrm{~h}$ posteriores a la exposición de PFD $(10 \mu \mathrm{M}, 100 \mu \mathrm{M}$ y $1.000 \mu \mathrm{M})$ o ALA $(5 \mathrm{mM})$. Para analizar la activación del factor transcripcional Nrf2, en diferentes tipos de células hepáticas, las HSC fueron tratadas con PFD $(100 \mu \mathrm{M}), \operatorname{MEN}(0,1$ $\mu \mathrm{M})$ o una combinación de ambos por 30', de la misma manera las células HepG2 fueron expuestas a ALA ( $5 \mathrm{mM})$, posterior al tiempo establecido se llevó a cabo la extracción de proteínas nucleares para el análisis por Western Blot. Finalmente para analizar el efecto de PFD en la generación de ROS inducida por MEN, las HSC fueron expuestas a $100 \mu \mathrm{M}$ de PFD y $0,1 \mu \mathrm{M}$ de MEN por 15 '. El nivel de ROS generado fue detectado mediante la conversión de 2',7'-diclorodihidrofluoresceina diacetato (DCFH-DA).

\section{Cultivo de células hepáticas}

Las HSC fueron aisladas a partir de secciones de hígado humano normal no aptos para el trasplante, mediante digestión con colagenasapronasa, además de ultracentrifugación sobre gradientes de Stractan y mantenidas en cultivo de monocapa en medio IMEM a 30\% FBS, 1\% Glutamax y $1 \%$ aminoácidos no esenciales a $37^{\circ} \mathrm{C}$ en atmósfera húmeda a $5 \% \mathrm{CO}_{2}$. El procedimiento para el aislamiento celular ha sido detalladamente descrito previamente ${ }^{30}$. Brevemente, las secciones de aproximadamente $50 \mathrm{~g}$ fueron disgregadas mecánicamente y cortadas con una hoja de bisturí hasta hacerla homogénea, posteriormente el macerado se transfirió a un matraz estéril que contiene $100 \mathrm{ml}$ de solución salina balanceada de Hank (HBSS, del inglés Hank's balanced salt solution. Life Technologies) sin Ca y Mg + 0,5\% de pronasa $(71,5 \mathrm{PUK} / \mathrm{mg}$. Calbiochem $)+0,05$ colagenasa (Tipo IV. Sigma) + DNasa $5 \mathrm{~g} / \mathrm{ml}+$ antibiótico antimicótico (Life Technologies) a $1 \%$ y se incubó a $37^{\circ} \mathrm{C}$ durante $30 \mathrm{~min}$. El tejido retenido en la gasa el cual no fue digerido, se trató nuevamente con solución HBSS reducida (pronasa $0,05 \%$ + colagenasa $0,05 \%$ ). Posteriormente la suspensión celular se dividió en varios tubos de 50 $\mathrm{ml}$ y se realizaron 5 lavados con HBSS reducida durante $7 \mathrm{~min}$ a $500 \mathrm{~g}$, al finalizar los lavados, todos los precipitados celulares se reunieron en un tubo con $25 \mathrm{ml}$ de HBSS reducida y se colocaron en 4 tubos sobre cuatro gradientes de Stractan (Cellsep; Larex Inc., MN) a diferente densidad y se centrifugó a $20.000 \mathrm{rpm}$ por $30 \mathrm{~min}$. Se separó la fase correspondiente a las células estelares hepáticas y se realizaron dos lavados con HBSS (sin enzimas), se descartó el sobrenadante y las células fueron resuspendidas en IMEM con 30\% de suero bovino fetal, a las 24 h se reemplazó el medio y las células adheridas fueron utilizadas para los experimentos entre los pases 3-7. Las HSC utilizadas mostraron características de miofibroblasto, lo que indica una completa activación. Las células HepG2 (No. AGT975345) se obtuvieron de la American Type Culture Collection (Manassas, VA), fueron mantenidas en cultivo de monocapa en medio DMEM al 8\% FBS, $1 \%$ Glutamax y $1 \%$ aminoácidos no esenciales a $37^{\circ} \mathrm{C}$ en atmósfera húmeda al $5 \% \mathrm{CO}_{2}$.

\section{Actividad metabólica, método de MTT}

La actividad metabólica celular fue determinada por el método de MTT basado en la conversión del bromuro de 3-(4,5-dimetil-tiazol-2-il)-2,5difenil-tetrazolio en azul formazán por la enzima succinato-deshidrogenasa, la tasa de conversión es proporcional al número de células metabólicamente activas. Cuatro horas previas a los tiempos analizados se añadió el MTT $(5 \mathrm{mg} / \mathrm{ml})$ al cultivo celular y se incubó a $37^{\circ} \mathrm{C}$, posteriormente se añadió la solución de $5 \% \mathrm{SDS} / 0,05 \mathrm{~N} \mathrm{HCl}$ por $20 \mathrm{~h}$ a $37^{\circ} \mathrm{C}$ y se cuantificó espectrofotométricamente a $579 \mathrm{~nm}$. Los resultados se expresan como la actividad metabólica relacionada al porcentaje de células viables. 


\section{RT-PCR cuantitativa}

El RNA total fue aislado utilizando el RNeasy Mini Kit (QIAGEN, CA) y retrotranscrito mediante la transcriptasa inversa MMLV (200 U) utilizando el sistema de síntesis Superscript y hexámeros aleatorios (Invitrogen-Life Technologies, CA). El cDNA fue tratado con DNAsa I (Invitrogen-Life Technologies, CA). Los productos retrotranscritos fueron amplificados mediante reacción en cadena de la polimerasa de tipo cuantitativa (qPCR) utilizando el TaqMan Universal PCR Master Mix y los oligonucleótidos específicos prediseñados (Applied Biosystems, Hammonton, NJ) para los genes GCLC, glutamil-cisteína sintetasa subunidad catalítica (NM_001498), GCLM, glutamil-cisteína sintetasa subunidad moduladora (NM_002061), HMOX1, hemoxigenasa 1 (NM_002133), SOD1, superóxido dismutasa 1 (NM_000454), IL6, interleucina 6 (NM_000600), MCP1, proteína quimiotáctica de monocitos 1 (NM_002982), TNF $\alpha$, factor de necrosis tumoral alfa (NM_000594), COL1A1, colágena tipo I alfa 1 (NM_000088), TGF- $\beta 1$, factor de crecimiento transformante beta 1 (NM_000660), TIMP1, inhibidor de metaloproteasas TIMP 1 (NM_003254) y GAPDH, gliceraldehído 3-fosfato deshidrogenasa (NM_002046). Los ciclos realizados fueron de 2 $\min / 50^{\circ} \mathrm{C}, 2 \mathrm{~min} / 95^{\circ} \mathrm{C}$, y 40 ciclos de $30 \mathrm{~s} / 95^{\circ} \mathrm{C}$ y $40 \mathrm{~s} / 60^{\circ} \mathrm{C}$ utilizando el equipo Rotor Gene-3000 (Corbett Life Science). Los resultados se indican como las veces de aumento o disminución de la expresión génica normalizado a los niveles del gen constitutivo gliceraldehído 3-fosfato deshidrogenasa (GAPDH) y relacionados con los niveles del gen correspondiente de la muestra control expresado como $2^{-\Delta \Delta \mathrm{Ct}}$.

\section{Contenido de glutatión}

Los niveles de GSH (glutatión reducido) y GSSG (glutatión oxidado) fueron determinados según el principio de reciclaje enzimático ${ }^{32}$, según las indicaciones del proveedor (Glutathione Colorimetric Detection Kit Arbor Assays, MI. USA).

\section{Análisis de Nrf2 por Western Blot}

Las fracciones celulares fueron aisladas como se ha descrito previamente ${ }^{28}$. Las células fueron tratadas con el tampón hipotónico para aislar la fase citosólica y un tampón hipertónico para aislar la fase nuclear. Posterior a la cuantificación proteica, $12 \mu \mathrm{g}$ de proteína nuclear fueron cargados en geles desnaturalizantes a 12\% de SDS-poliacrilamida y transferidos a membrana de PVDF 0,2 $\mu \mathrm{m}$ (Bio-Rad. CA). Posterior a la detección del Nrf2 (anti-Nrf2 1:200) se digitalizaron las bandas y se eliminó (stripping) el anticuerpo Nrf2 utilizando el tampón Western Blot Stripping Buffer según indicaciones del proveedor (Santa Cruz, CA), posteriormente se detectó (reprobe) la fibrilarina (anti-fibrilarina 1:500). La densidad relativa de las bandas generadas con el sistema de peroxidasa se analizó digitalmente utilizando el programa Kodak ID Image Analysis, los resultados se expresaron como Unidades Relativas de Área (U.R.A.).

\section{Detección del nivel intracelular de ROS}

La generación intracelular de ROS se detectó mediante el uso de la conversión de 2,7-diclorodihidrofluoresceina diacetato (DCFH-DA) por esterasas en el derivado fluorescente correspondiente. La densidad relativa de la fluorescencia emitida por las células de diez campos al azar, fue cuantificado digitalmente utilizando el programa Kodak ID Image Analysis, los valores se expresaron como Unidades Relativas de Área (U.R.A.).

\section{Análisis estadístico}

Los resultados se expresan como la media \pm desviación estándar. El análisis estadístico se realizó mediante el análisis de varianza a dos colas, cuando el valor de $p$ fue significativo $(\mathrm{p} \leq 0,05)$ mediante la prueba de Duncan.

\section{Resultados}

\section{PFD reduce la actividad metabólica celular basal e inducida por PDGF}

Mediante el método de MTT se cuantificó la tasa de actividad metabólica mitocondrial, la cual es proporcional al número de células viables, lo que indica el grado de proliferación celular. Las HSC del grupo control presentaron el crecimiento característico para el período analizado. Este efecto se redujo por el pre-tratamiento con PFD a partir de $100 \mu \mathrm{M}$ (Figura 1A). El PDGF $10 \mathrm{ng} / \mathrm{ml}$ por 24 $\mathrm{h}$ aumentó el crecimiento de las HSC vs control. Este efecto fue disminuido significativamente por el pre-tratamiento con PFD $100 \mu \mathrm{M}$. El MEN 0,1 $\mu \mathrm{M}$ no modificó la actividad metabólica celular (Figura 1B). 
A

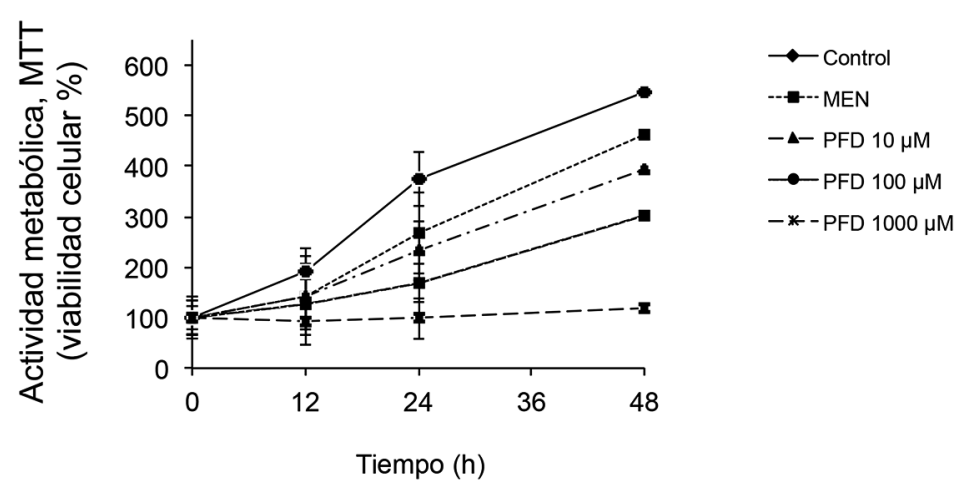

B

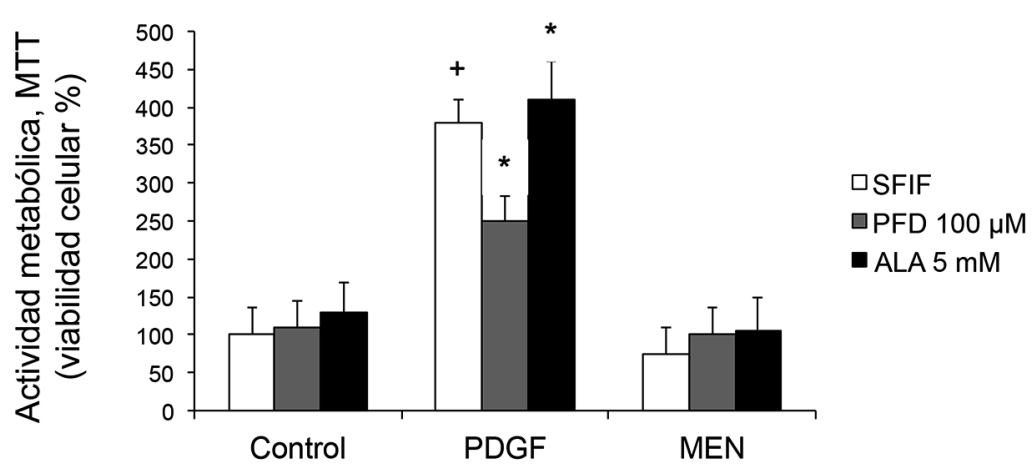

Figura 1. Efecto de la PFD en la actividad metabólica mitocondrial en células HSC. A) Tiempo acción de la actividad metabólica mitocondrial en células tratadas con PFD y MEN a las 12, 24 y 48 h; B) Efecto de PFD $100 \mu$ M y ALA 5 mM en la actividad metabólica mitocondrial inducida por PDGF $(10 \mathrm{ng} / \mathrm{ml})$ y $\operatorname{MEN}(0,1 \mu \mathrm{M})$ por $24 \mathrm{~h}$. La actividad metabólica celular fue determinada por la reducción de la sal de tetrazolio a azul formazán por la succinato deshidrogenasa mitocondrial (ensayo de MTT). Los valores representan la media \pm la desviación estándar de tres experimentos independientes y por triplicado de la actividad metabólica relacionada al porcentaje de células viables. ${ }^{+} p \leq 0,05$ comparada con PDGF. ${ }^{*} p \leq 0,05$ comparada con el grupo control suplementado con medio de cultivo SFIF (medio de cultivo libre de suero bovino fetal e insulina). PFD, 5-metil1-fenil-2-(1H)-piridona o pirfenidona. MEN, 2-metil-1.4-naftoquinona o menadiona. ALA, ácido alfa-lipoico. PDGF, factor de crecimiento derivado de plaquetas.

\section{PFD induce la expresión de genes antioxidantes $y$ los niveles de GSH}

La PFD $100 \mu \mathrm{M}$ per se incrementó principalmente a las $12 \mathrm{~h}$ la expresión del mRNA $\left(2^{-\Delta \Delta C t}\right)$ de los genes antioxidantes GCLM $(5,9)$, GCLC $(2,5)$ y HMOX1 $(3,4)$, pero no la SOD1 $(1,2)$. El ALA $5 \mathrm{mM}$ indujo la misma respuesta, GCLM $(13,0)$, $\operatorname{GCLC}(10,7), \operatorname{HMOX} 1(3,3)$ entre las 8 y las $12 \mathrm{~h}$ (Figura 2A), esto correlaciona con el incremento del nivel de GSH en las células tratadas con PFD o ALA (Figura 3). En cambio, cuando las HSC fueron expuestas tanto a PFD como a MEN (Tabla 1), la expresión de GCLC y GCLM fue menor vs las HSC tratadas sólo con MEN, la expresión de la HMOX1 y SOD presentaron la misma tendencia, sin embargo, esta disminución no indujo cambios estadísticamente significativos en comparación con las células tratadas con PFD. 
A

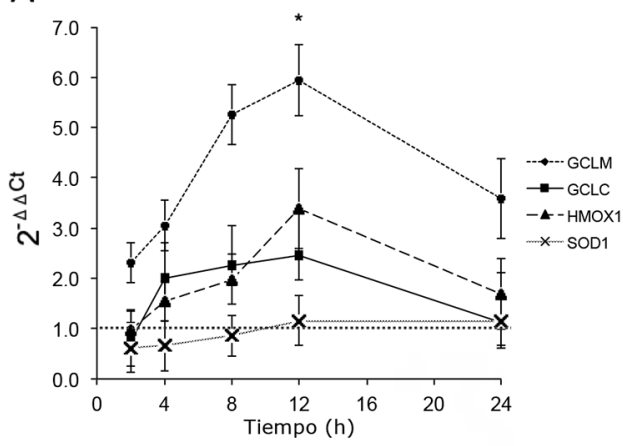

C

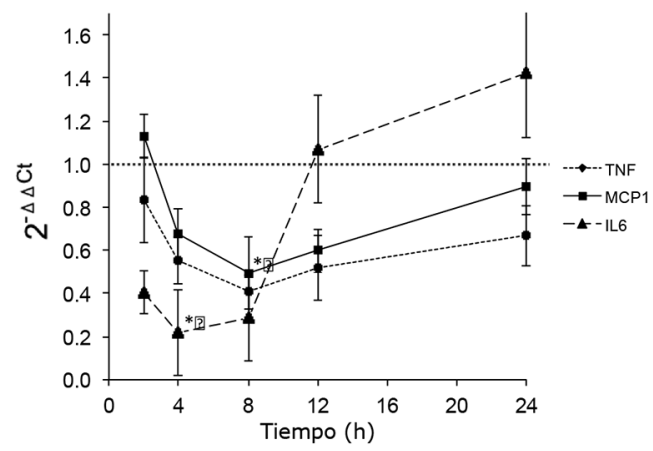

B

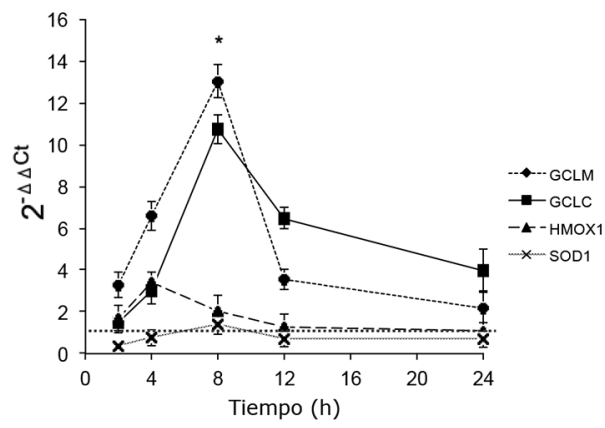

D

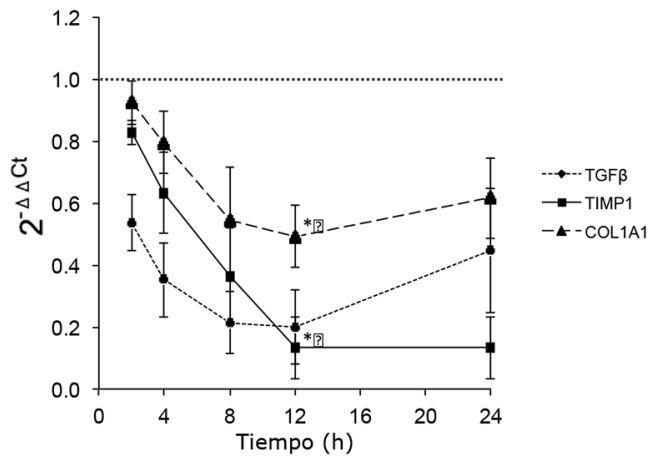

Figura 2. Modulación de la expresión génica de acuerdo a las categorías antioxidante, inflamatoria y fibrogénica en HSC. Cuantificación de la expresión del RNAm de genes: A) antioxidantes inducida por PFD $100 \mu \mathrm{M}$; B) antioxidantes inducida por ALA $5 \mathrm{mM}$. Las categorías C) inflamatoria y D) fibrogénica moduladas por PFD $100 \mu \mathrm{M}$, a las 2, 4, 8, 12 y 24 h. Las gráficas representan [sobre-expresión $(>1)$, expresión basal del control $(=1)$, disminución en la expresión $(<1)$ ] la media \pm desviación estándar de las veces de cambio $\left(2^{-\Delta \Delta c t}\right)$ con respecto al grupo control $=1$ (línea gris punteada). La expresión del RNAm de los genes analizados fue normalizada a los niveles de expresión del gen constitutivo gliceraldehído 3-fosfato deshidrogenasa (GAPDH). ${ }^{*} p \leq 0,05$ en comparación con el grupo control. $n=3$. GCLC, glutamil-cisteína sintetasa subunidad catalítica. GCLM, glutamil-cisteína sintetasa subunidad moduladora. HMOX1, hemoxigenasa 1. SOD1, superóxido dismutasa 1, IL6, interleucina 6. MCP1, proteína quimiotáctica de monocitos 1. TNF $\alpha$, factor de necrosis tumoral alfa. COL1A1, colágena tipo I alfa 1 . TGF- $\beta 1$, factor de crecimiento transformante beta 1. TIMP1, inhibidor de metaloproteasas TIMP 1.

\section{PFD reduce la respuesta inflamatoria $y$ fibrogénica basal e inducida por estrés oxidativo en las HSC}

Posterior al tratamiento con PFD $100 \mu \mathrm{M}$, se observó la disminución en los niveles basales de la expresión del mRNA $\left(2^{-\Delta \Delta C t}\right)$ de los genes proinflamatorios IL6 $(0,22,4 \mathrm{~h}), \mathrm{MCP} 1(0,50,8$ h) y TNF $\alpha(0,41,8 \mathrm{~h})$ (Figura 2C), así como de los genes profibrogénicos COL1A1 $(0,49)$, TGF- $\beta 1(0,20)$ y el TIMP1 $(0,13)$ comparándolos con el grupo control $(=1)$ a las $12 \mathrm{~h}$ (Figura $2 \mathrm{D})$, esta tendencia también se observó en células expuestas a PFD $100 \mu \mathrm{M}+\mathrm{MEN}$ 0,1 $\mu \mathrm{M}$ (Tabla 1).

\section{Efecto de la PFD en la importación nuclear del factor de transcripción Nrf2}

La exposición de las HSC a PFD $100 \mu \mathrm{M}$ o MEN 0,1 $\mu \mathrm{M}$ (Figura 4A) mostró un incremento 


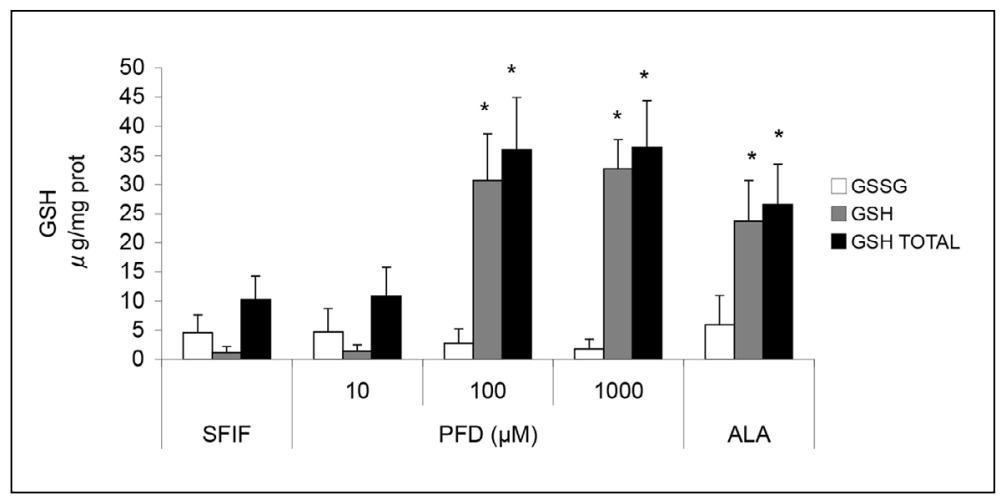

Figura 3. Efecto de PFD en los niveles de glutatión en las HSC. Los niveles de glutatión reducido (GSH), glutatión oxidado (GSSH) y GSH total (Total) fueron cuantificados en las HSC tratadas con PFD $10 \mu \mathrm{M}, 100 \mu \mathrm{M}$ y $1.000 \mu \mathrm{M}$ así como con ALA $5 \mathrm{mM}$ por $24 \mathrm{~h}$. ${ }^{*} p \leq 0,05$ comparada con el grupo control (SFIF). $\mathrm{n}=3$. SFIF, medio de cultivo libre de suero bovino fetal e insulina. PFD, 5-metil1-fenil-2-(1H)-piridona o pirfenidona. ALA, ácido alfa-lipoico.

Tabla 1. Análisis de la expresión génica inducida por la PFD en las HSC expuestas a MEN

\begin{tabular}{|c|c|c|c|c|c|c|}
\hline \multirow[t]{2}{*}{$\begin{array}{l}\text { Categoría } \\
\text { funcional }\end{array}$} & \multirow[t]{2}{*}{ Nombre } & \multirow[t]{2}{*}{ ID } & \multicolumn{4}{|c|}{$\begin{array}{c}\text { Menadiona (MEN) } \\
\text { PFD }\end{array}$} \\
\hline & & & & $10 \mu \mathrm{M}$ & $100 \mu \mathrm{M}$ & $1.000 \mu \mathrm{M}$ \\
\hline Antioxidante & $\begin{array}{l}\text { Glutamil-cisteína sintetasa subunidad catalítica } \\
\text { Glutamil-cisteína sintetasa subunidad moduladora } \\
\text { Hemoxigenasa } 1 \\
\text { Superóxido dismutasa } 1\end{array}$ & $\begin{array}{l}\text { GCLC } \\
\text { GCLM } \\
\text { HMOX1 } \\
\text { SOD1 }\end{array}$ & $\begin{array}{l}2,35 \pm 0,2 \\
2,52 \pm 0,4 \\
1,93 \pm 0,1 \\
1,41 \pm 0,2\end{array}$ & $\begin{array}{l}1,57 \pm 0,6 \\
1,61 \pm 0,2 \\
1,53 \pm 0,3 \\
1,12 \pm 0,4\end{array}$ & $\begin{array}{l}1,89 \pm 0,9 \\
1,96 \pm 0,3 \\
1,11 \pm 0,4 \\
1,29 \pm 0,4\end{array}$ & $\begin{array}{l}1,92 \pm 0,2 \\
2,00 \pm 0,4 \\
0,97 \pm 0,3 \\
1,01 \pm 0,5\end{array}$ \\
\hline Inflamatoria & $\begin{array}{l}\text { Interleucina } 6 \\
\text { Proteína quimiotáctica de monocitos } 1 \\
\text { Factor de necrosis tumoral alfa }\end{array}$ & $\begin{array}{l}\text { IL6 } \\
\text { MCP1 } \\
\text { TNF } \alpha\end{array}$ & $\begin{array}{l}1,53 \pm 0,3 \\
1,40 \pm 0,1 \\
1,43 \pm 0,2\end{array}$ & $\begin{array}{l}1,42 \pm 0,5 \\
1,29 \pm 0,3 \\
1,32 \pm 0,7\end{array}$ & $\begin{array}{l}1,06 \pm 0,1 \\
1,16 \pm 0,2 \\
1,08 \pm 0,3\end{array}$ & $\begin{array}{l}1,06 \pm 0,3 \\
0,70 \pm 0,1 \\
1,06 \pm 0,5\end{array}$ \\
\hline Fibrogénica & $\begin{array}{l}\text { Colágena tipo I alfa } 1 \\
\text { Factor de crecimiento transformante beta } 1 \\
\text { Inhibidor de metaloproteasas TIMP } 1\end{array}$ & $\begin{array}{l}\text { COL1A1 } \\
\text { TGF- } \beta 1 \\
\text { TIMP1 }\end{array}$ & $\begin{array}{l}1,92 \pm 0,2 \\
1,53 \pm 0,6 \\
1,50 \pm 0,4\end{array}$ & $\begin{array}{l}1,82 \pm 0,5 \\
1,33 \pm 0,3 \\
1,09 \pm 0,3\end{array}$ & $\begin{array}{l}1,12 \pm 0,1 \\
1,33 \pm 0,1 \\
1,07 \pm 0,1\end{array}$ & $\begin{array}{l}0,99 \pm 0,4 \\
1,27 \pm 0,2 \\
0,98 \pm 0,3\end{array}$ \\
\hline
\end{tabular}

Expresión génica inducida por PFD $(10,100$ y $1.000 \mu \mathrm{M})$ de manera dosis dependiente de acuerdo a las categorías funcionales antioxidante, inflamatoria y fibrogénica en células expuestas a MEN $(0,1 \mu \mathrm{M})$ por $8 \mathrm{~h}$. Los valores representan la media \pm desviación estándar de las veces de cambio $\left(2^{-\Delta \Lambda c t}\right)$ con respecto al grupo control $(=1)$. La expresión del RNAm de los genes analizados fue normalizada a los niveles de expresión del gen constitutivo gliceraldehído 3-fosfato deshidrogenasa (GAPDH). $n=3$. PFD, 5-metil-1-fenil-2-(1H)-piridona o pirfenidona. MEN, 2-metil-1.4-naftoquinona o menadiona.

del nivel de Nrf2 en el núcleo celular comparado con el control, las HSC tratadas con PFD 100 $\mu \mathrm{M}+\mathrm{MEN} 0,1 \mu \mathrm{M}$ mostraron un menor nivel de $\mathrm{Nrf} 2$ en este compartimento celular. La Figura 4B muestra un incremento significativo del nivel de Nrf2 inducido por ALA $5 \mathrm{mM}$ vs grupo control, lo que confirma la activación de este sistema en dos tipos de células hepáticas.

\section{PFD disminuye la generación de ROS inducida por el MEN}

La generación intracelular de ROS se detectó por la conversión de 2',7'-dichlorodihydrofluo- rescein diacetato (DCFH-DA) en respuesta a MEN $0,1 \mu \mathrm{M}$ en presencia o ausencia de PFD $100 \mu \mathrm{M}$. Como se observa en la Figura 5, la PFD reduce notablemente la generación de ROS intracelular inducida por este sistema pro-oxidante.

\section{Discusión}

La PFD mostró propiedades anti-inflamatorias y anti-fibróticas tanto a nivel basal como en respuesta al estrés oxidativo en cultivo primario de HSC humanas, lo cual se asocia a la activación 
A
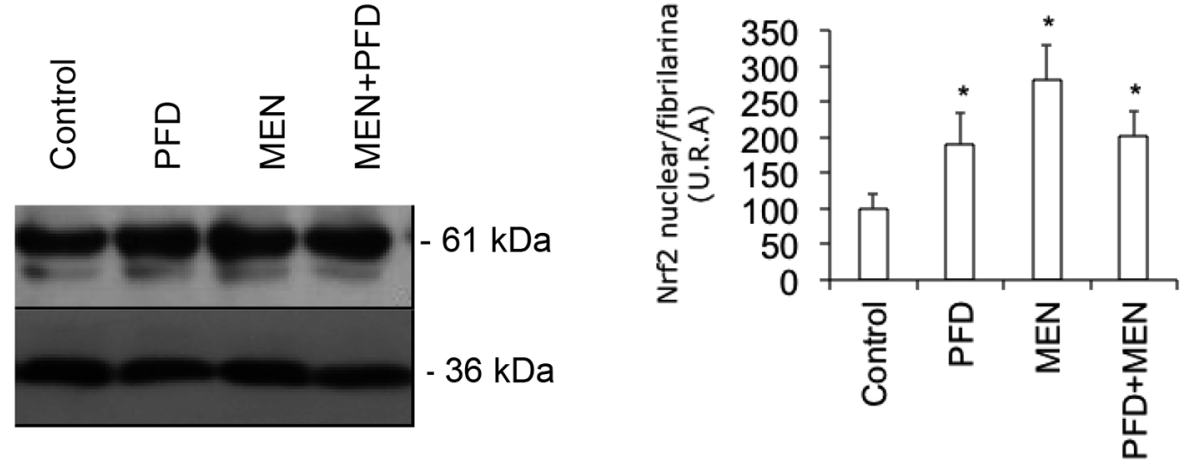

B
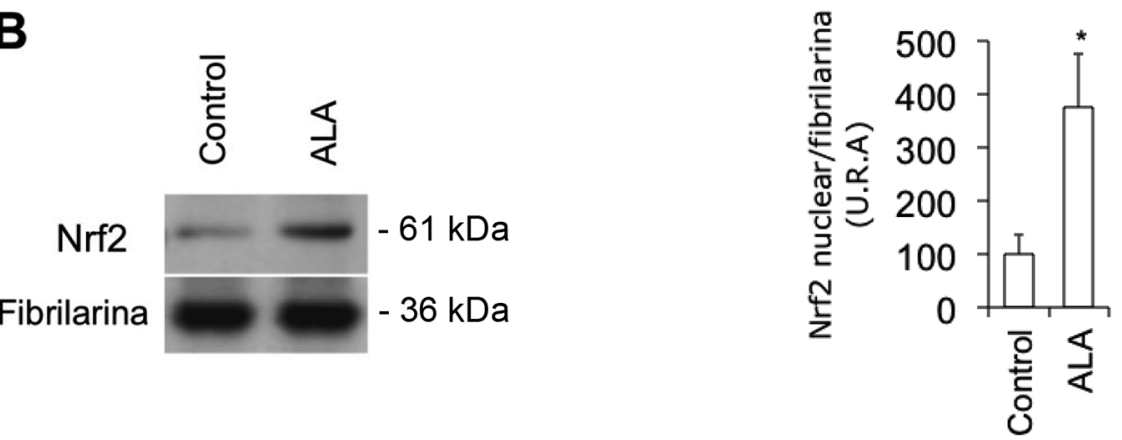

Figura 4. Efecto de la PFD en la traslocación nuclear del factor transcripcional Nrf2. A) Las HSC cultivadas en medio SFIF fueron expuestas a $100 \mu \mathrm{M}$ de PFD y 0,1 $\mu \mathrm{M}$ de MEN por 30'. Las imágenes son representativas de los resultados de Western Blot de la fracción nuclear $(12 \mu \mathrm{g}), \mathrm{n}=3$; B) Las células HepG2 fueron expuestas a $5 \mathrm{mM}$ de ALA por 30', las imágenes corresponden a la fracción nuclear $(15 \mu \mathrm{g}), \mathrm{n}=3$. Los resultados son representativos de tres experimentos independientes. Las gráficas resumen los resultados de dichos experimentos, donde los valores representan la media \pm desviación estándar de las Unidades Relativas de Área (U.R.A.). ${ }^{*} \mathrm{p} \leq 0,05$ comparada con el grupo control (SFIF).

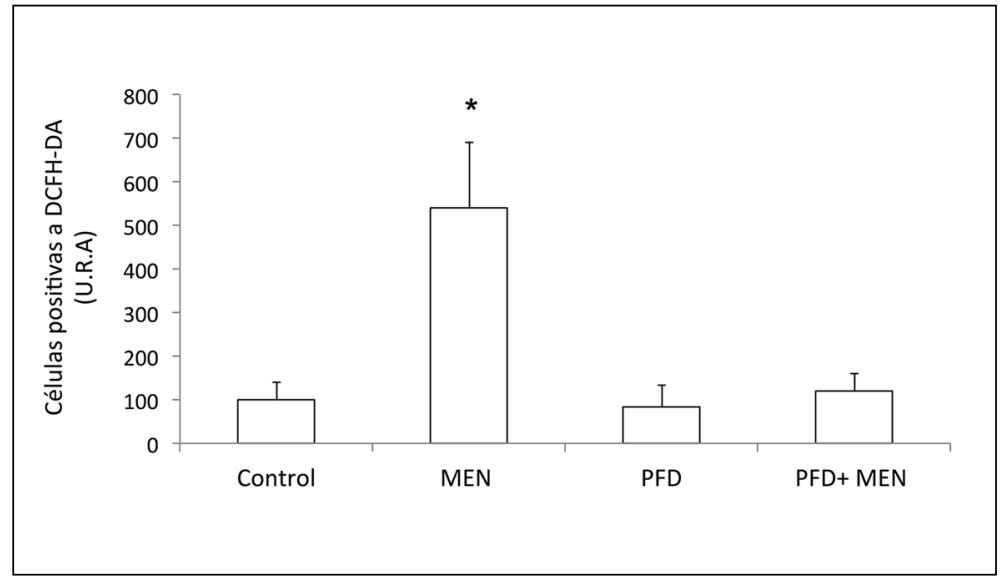

Figura 5. Efecto de la PFD en el nivel de ROS intracelular inducido por MEN. Las HSC fueron expuestas a $100 \mu \mathrm{M}$ de PFD y $0,1 \mu \mathrm{M}$ de MEN por 15'. El nivel de ROS generado fue detectado mediante la conversión de 2',7'-dichlorodihydrofluorescein diacetato (DCFH-DA). La gráfica resume los resultados derivados de la observación de diez campos microscópicos distintos para cada uno de tres experimentos independientes. Los valores se expresaron como Unidades Relativas de Área (U.R.A.) y representan la media \pm SD. ${ }^{*} p<0,05$. 
del sistema antioxidante. En cuanto a los efectos antioxidantes, se ha observado que la PFD inhibe la lipoperoxidación ${ }^{29}$ mediante la inhibición de la enzima óxido nítrico sintetasa inducible (iNOS) ${ }^{30}$, lo que reduce la generación de ROS y disminuye la señalización para la activación de las HSC ${ }^{31}$. Además nuestros resultados muestran un claro incremento del nivel de GSH y del mRNA de los genes GCLC, GCLM y HMOX1 por la PFD, respuesta similar a la producida por el ALA. Sin embargo, no se observó un incremento en la SOD1. Así mismo el MEN incrementó la expresión del mRNA de estos genes y de SOD1, en este caso no como una inducción pro-antioxidante, sino como una respuesta protectora por el incremento de ROS. Esto es relevante, ya que no hay reportes donde se indique que la PFD induce per se la activación de sistemas antioxidantes. La activación de la mayoría de los genes antioxidantes es dirigida por el factor transcripcional $\mathrm{Nrf2}^{32-35}$; sin embargo, en el caso de SOD1 es probable que esta enzima requiera de la co-activación de factores transcripcionales sensibles al radical $\mathrm{O}_{2} \cdot-$ que comprenden eventos intracelulares complejos e involucran múltiples elementos de regulación positiva y negativa ${ }^{36}$.

En condiciones normales, varios tipos de células expresan Nrf2 constitutivamente y es reprimido por el inhibidor Keap1 en el citoplasma, durante procesos de oxidación este inhibidor sufre cambios conformacionales y libera al Nrf2 para que éste sea transportado al núcleo ${ }^{32,37-39}$, lo cual pudimos confirmar al tratar células HepG2 con el ALA, donde el Nrf2 nuclear incrementó con respecto a las células no tratadas.

El tratamiento con PFD o PFD+MEN mostró un incremento significativo de Nrf2 en el núcleo celular, este fenómeno puede llevarse a cabo por varias vías, como el paso de Nrf2 del citoplasma al núcleo inmediatamente después de la traducción de la proteína, por reorganización de $\mathrm{Nrf} 2$ en el núcleo donde se facilita su disociación de Keap1 cerca de la membrana nuclear, o posiblemente como una disminución en el proceso de degradación de Nrf2. Por lo tanto, la promoción de este mecanismo o la intervención de varios de ellos parecen ser los responsables de mantener un mayor nivel de Nrf2 nuclear. Aún no se entiende si los mecanismos activados por la combinación de PFD+MEN operan de manera independiente o en forma coordinada, sin embargo, se plantea la hipótesis de que este efecto está relacionado a la interacción bioquímica de ambos compuestos y la participación de enzimas de fase I para el metabolismo de xenobióticos ${ }^{40,41}$. Está comprobado que enzimas con capacidad reductora, como la citocromo $\mathrm{P} 450$ reductasa y la NADH-ubiquinona oxidorreductasa metabolizan quinonas, en este caso la MEN, por reacciones de reducción de un electrón, las semiquinonas inestables resultantes pueden entrar fácilmente en un ciclo redox cuando el oxígeno está presente, induciendo que se genere nuevamente la quinona, con la generación concomitante de $\mathrm{ROS}^{42}$, sin embargo, la generación de ROS puede ser disminuida por PFD al reducir la cantidad de ROS como se pudo observar por la conversión de DCFH-DA. Además se observó que el incremento en la cantidad de ROS inducida por MEN activa enzimas de la fase II, como la GCLC y la GCLM, las cuales son reguladas por $\mathrm{Nrf} 2$, lo que demuestra que, en las HSC tratadas con PFD+MEN donde el nivel de Nrf2 importado al núcleo es menor que en las HSC tratadas sólo con MEN, el PFD tiene un efecto regulador en la activación de mediadores antioxidantes. Por lo tanto, el efecto protector de la PFD podría actuar por vías diferentes, la primera por inhibición directa de ROS, y la otra por la inducción de una incrementada importación de Nrf2 al núcleo lo que conlleva al incremento transcripcional de genes que codifican para enzimas antioxidantes con efectos protectores, sin embargo, se requiere analizar la interacción química entre ambos compuestos previo a la activación de Nrf2.

Con respecto a la proliferación celular, se observó que el MEN no incrementa la proliferación de las HSC, por lo tanto, durante daño hepático crónico, el estrés oxidativo no induce la proliferación, pero si la formación y liberación de citocinas y factores del crecimiento, y son éstos los que promueven la proliferación celular. Además la PFD disminuyó la proliferación (analizado como actividad mitocondrial) inducida por el PDGF o el MEN, lo cual correlaciona con la reducción de la síntesis basal e inducida de las citocinas IL6, MCP1 y TNF $\alpha$, lo que se asocia con una relevante actividad anti-inflamatoria, ya que estos mediadores están relacionados con el infiltrado inflamatorio en el tracto portal durante la hepatitis crónica. Además, la secreción de citocinas proinflamatorias y quimiocinas por las HSC, contribuye no sólo al reclutamiento sino a la activación de nuevos monocitos en el sitio de la lesión. La inflamación 
Efecto de pirfenidona en el nivel de glutatión en células estelares hepáticas - J. Macias-Barragan et al

prolongada conduce a un cambio progresivo en el tipo de células presentes en el lugar del daño, que se caracteriza por la cicatrización del tejido posterior al proceso inflamatorio, es aquí donde los mediadores profibrogénicos son activados. Con respecto a este proceso se observó que la PFD disminuyó la expresión basal e inducida por el MEN de COL1A1, TGF- $\beta 1$ y TIMP1, los cuales juegan un papel importante en el desarrollo de la fibrosis hepática, estableciendo un ciclo autócrino el cual conlleva a la proliferación de los fibroblastos y su diferenciación en miofibroblastos secretores de colágeno. Estos mediadores son reconocidos como los principales conductores hacia el fenotipo fibrótico. Se ha reportado que el TGF- $\beta 1$ disminuye la expresión y la actividad de enzimas antioxidantes ${ }^{43-45}$, y de forma inversa la activación de Nrf2 puede suprimir la expresión del TGF- $\beta 1$ y la activación de las HSC ${ }^{46-49}$, efectos observados con el tratamiento con PFD. Por lo tanto, la modulación del sistema antioxidante está íntimamente relacionada con la regulación de la activación de las $\mathrm{HSC}^{50-52}$, sin olvidar que el lapso y nivel de daño en el microambiente hepático puede determinar la dirección hacia la fibrólisis o la fibrogénesis tisular.

En conclusión, la PFD promueve el potencial antioxidante endógeno de las HSC al inducir la expresión de genes antioxidantes y del GSH ya que es probable que las principales acciones terapéuticas de la PFD en condiciones de daño hepático crónico parecen ser debidas a la interacción entre sus efectos antifibróticos así como la modulación de la actividad anti-inflamatoria y anti-oxidante en un contexto caracterizado por la inhibición de la activación de las HSC.

\section{Referencias}

1. Pinzani M, Macias-Barragan J. Update on the pathophysiology of liver fibrosis. Expert Rev Gastroenterol Hepatol 2010; 4: 459-72.

2. Hancock R, Bertrand HC, Tsujita T, Naz S, El-Bakry A, Laoruchupong J, et al. Peptide inhibitors of the Keap1Nrf2 protein-protein interaction. Free Radic Biol Med 2012; 52 (2): 444-51.

3. Itoh K, Wakabayashi N, Katoh Y, Ishii T, Igarashi K, Engel JD, et al. Keap1 represses nuclear activation of antioxidant responsive elements by Nrf2 through binding to the amino-terminal Neh2 domain. Genes Dev 1999; 13 (1): 76-86
4. Kobayashi A, Kang MI, Watai Y, Tong KI, Shibata T, Uchida K, et al. Oxidative and electrophilic stresses activate Nrf2 through inhibition of ubiquitination activity of Keap1. Mol Cell Biol 2006; 26 (1): 221-9.

5. Nguyen T, Sherratt PJ, Nioi P, Yang CS, Pickett CB. Nrf2 controls constitutive and inducible expression of AREdriven genes through a dynamic pathway involving nucleocytoplasmic shuttling by Keap1. J Biol Chem 2005; 280 (37): 32485-92.

6. Dinkova-Kostova AT, Holtzclaw WD, Cole RN, Itoh K, Wakabayashi N, Katoh Y, et al. Proc Natl Acad Sci 2002; 99: 11908-13.

7. Wakabayashi N, Dinkova-Kostova AT, Holtzclaw WD, Kang MI, Kobayashi A, Yamamoto, et al. Proc Natl Acad Sci USA 2004; 101: 2040-45.

8. Aleksunes LM, Manautou JE. Emerging role of Nrf2 in protecting against hepatic and gastrointestinal disease. Toxicol Pathol 2007; 35 (4): 459-73.

9. Varì R, D'Archivio M, Filesi C, Carotenuto S, Scazzocchio B, Santangelo C, et al. Protocatechuic acid induces antioxidant/detoxifying enzyme expression through JNK-mediated Nrf2 activation in murine macrophages. J Nutr Biochem 2011; 22 (5): 409-17.

10. Xu C, Yuan X, Pan Z, Shen G, Kim JH, Yu S, et al. Mechanism of action of isothiocyanates: the induction of AREregulated genes is associated with activation of ERK and JNK and the phosphorylation and nuclear translocation of Nrf2. Mol Cancer Ther 2006; 5 (8): 1918-26.

11. Yoshida K, Matsuzaki K, Mori S, Tahashi Y, Yamagata $\mathrm{H}$, Furukawa F, et al. Transforming growth factor-beta and platelet-derived growth factor signal via c-Jun Nterminal kinase-dependent $\operatorname{Smad} 2 / 3$ phosphorylation in rat hepatic stellate cells after acute liver injury. Am J Pathol 2005; 166: 1029-39.

12. Schnabl B, Bradham C, Bennett B, Manning A, Stefanovic B, Brenner D. TAK1/JNK and p38 have opposite effects on rat hepatic stellate cells. Hepatology 2001; 34: 953-63.

13. Oakley F, Meso M, Iredale JP, Green K, Marek CJ, Zhou $\mathrm{X}$, et al. Inhibition of inhibitor of kappaB kinases stimulates hepatic stellate cell apoptosis and accelerated recovery from rat liver fibrosis. Gastroenterology 2005; 128: 108-20.

14. Dietrich N, Thastrup J, Holmberg C, Gyrd-Hansen M, Fehrenbacher N, Lademann U, et al. JNK2 mediates TNF-induced cell death in mouse embryonic fibroblasts via regulation of both caspase and cathepsin protease pathways. Cell Death Differ 2004; 11: 301-13.

15. Köhle C, Bock KW. Coordinate regulation of Phase I and II xenobiotic metabolisms by the Ah receptor and Nrf2. Biochem Pharmacol 2007; 73 (12): 1853-62. 
16. Shen G, Kong AN. Nrf2 plays an important role in coordinated regulation of Phase II drug metabolism enzymes and Phase III drug transporters. Biopharm Drug Dispos 2009; 30 (7): 345-55.

17. Königsberg M. Nrf2: la historia de un nuevo factor de transcripción que responde a estrés oxidativo. Revista de Educación Bioquímica 2007; 26 (1): 18-25.

18. Li W, Khor TO, Xu C, Shen G, Jeong WS, Yu S, et al. Activation of Nrf2-antioxidant signaling attenuates NFkappaB-inflammatory response and elicits apoptosis. Biochem Pharmacol 2008; 76 (11): 1485-9.

19. Zamara E, Novo E, Parola M. Oxidative stress and liver fibrosis: from liver injury to the modulation of cell signaling and response. En: Liver Diseases: Biochemical Mechanisms and New Therapeutic Insights. Ali S, Mann DA, Friedman SL. Enfield NH (Eds). Science Publishers. Volumen 1, EUA, 2004: 93-114.

20. Parola M, Robino G. Oxidative stress-related molecules and liver fibrosis. J Hepatol 2001; 35: 297-306.

21. Bedossa P, Houglum K, Trautwein C, Holstege A, Chojkier M. Stimulation of collagen $\alpha$ (I) gene expression is associated with lipid peroxidation in hepatocellular injury. A link to tissue fibrosis? Hepatology 1994; 19: 1262-71.

22. Sass G, Barikbin R, Tiegs G. The Multiple Functions of Heme Oxygenase-1 in the Liver. Z Gastroenterol 2012; 50 (1): 34-40.

23. Liu RM, Gaston Pravia KA. Oxidative stress and glutathione in TGF-beta-mediated fibrogenesis. Free Radic Biol Med 2010; 48 (1): 1-15.

24. Aldaba-Muruato LR, Moreno MG, Shibayama M, Tsutsumi V, Muriel P. Protective effects of allopurinol against acute liver damage and cirrhosis induced by carbon tetrachloride: Modulation of NF-kB, cytokine production and oxidative stress. Biochim Biophys Acta 2011; 1820 (2): 65-75.

25. Schaefer CJ, Ruhrmund DW, Pan L, Seiwert SD, Kossen $\mathrm{K}$. Antifibrotic activities of pirfenidone in animal models. Eur Respir Rev 2011; 20 (120): 85-97.

26. Richeldi L, Yasothan U, Kirkpatrick P. Pirfenidone. Nat Rev Drug Discov 2011; 10 (7): 489-90.

27. Mitani Y, Sato K, Muramoto Y, Karakawa T, Kitamado $\mathrm{M}$, Iwanaga T, et al. Superoxide scavenging activity of pirfenidone-iron complex. Biochem Biophys Res Commun 2008; 372 (1): 19-23.

28. Salazar-Montes A, Ruiz-Corro L, López-Reyes A, Castrejón-Gómez E, Armendáriz-Borunda J. Potent antioxidant role of pirfenidone in experimental cirrhosis. Eur J Pharmacol 2008; 595 (1-3): 69-77.

29. Huerta-Olvera SG, Macías-Barragán J, Ramos-Márquez ME, Armendáriz-Borunda J, Díaz-Barriga F, Siller-López
F. Alpha-lipoic acid regulates heme oxygenase gene expression and nuclear Nrf2 activation as a mechanism of protection against arsenic exposure in HepG2 cells. Environ Toxicol Pharmacol 2010; 29 (2): 144-9.

30. Casini A, Pinzani M, Milani S, Grappone C, Galli G, Jezequel A, et al. Regulation of extracellular matrix synthesis by transforming growth factor beta 1 in human fat-storing cells. Gastroenterology 1993; 105: 24553.

31. Tada H, Shiho O, Kuroshima K, Koyama M, Tsukamoto K. An improved colorimetric assay for interleukin 2. J Immunol Methods 1986; 93 (2): 157-65.

32. Tietze F. Enzymic method for quantitative determination of nanogram amounts of total and oxidized glutathione: applications to mammalian blood and other tissues. Anal Biochem 1969; 27 (3): 502-22.

33. Sun Z, Zhang S, Chan JY, Zhang DD. Keapl controls postinduction repression of the Nrf2-mediated antioxidant response by escorting nuclear export of Nrf2. Mol Cell Biol 2007; 27 (18): 6334-49.

34. Misra H, Rabideau C. Pirfenidone inhibits NADPHdependent microsomal lipid peroxidation and scavenges hydroxyl radicals. Molec Cell Biochem 2000; 204: 11926.

35. Saleh D, Barnes PJ, Giaid A. Increased production of the potent oxidant peroxynitrite in the lungs of patients with idiopathic pulmonary fibrosis. Am J Respir Crit Care Med 1997; 155: 1763-69.

36. Milani P, Gagliardi S, Cova E, Cereda C. SOD1 Transcriptional and Posttranscriptional Regulation and Its Potential Implications in ALS. Neurol Res Int 2011; 458427.

37. Novo E, Parola M. Redox mechanisms in hepatic chronic wound healing and fibrogenesis. Fibrogenesis Tissue Repair 2008; 13; 1 (1): 5.

38. Itoh K, Mochizuki M, Ishii Y, Ishii T, Shibata T, Kawamoto $\mathrm{Y}$, et al. Transcription factor Nrf2 regulates inflammation by mediating the effect of 15-deoxyDelta(12,14)-prostaglandin j(2). Mol Cell Biol 2004; 24 (1): 36-45.

39. Jaiswal AK. Nrf2 signaling in coordinated activation of antioxidant gene expression. Free Radic Biol Med 2004; 36 (10): 1199-207.

40. Lee JM, Johnson JA. An important role of Nrf2-ARE pathway in the cellular defense mechanism. J Biochem Mol Biol 2004; 37 (2): 139-43.

41. Zhang D, Hannink M. Distinct cysteine residues in Keap1 are required for Keap1-dependent ubiquitination of Nrf2 and for stabilization of Nrf2 by chemopreventive agents and oxidative stress. Mol Cell Biol 2003; 23 (22): 8137-51. 
Efecto de pirfenidona en el nivel de glutatión en células estelares hepáticas - J. Macias-Barragan et al

42. Criddle DN, Gillies S, Baumgartner-Wilson HK, Jaffar M, Chinje EC, Passmore S, et al. Menadione-induced reactive oxygen species generation via redox cycling promotes apoptosis of murine pancreatic acinar cells. J Biol Chem 2006; 281 (52): 40485-92.

43. Numazawa S, Yoshida T. Nrf2-dependent gene expressions: a molecular toxicological aspect. J Toxicol Sci 2004; 29 (2): 81-9.

44. Kang KW, Park EY, Kim SG. Activation of CCAAT/enhancer-binding protein beta by 2'-amino-3'-methoxyflavone (PD98059) leads to the induction of glutathione S-transferase A2. Carcinogenesis 2003; 24 (3): 475-82.

45. Levonen A, Landar A, Ramachandran A, Ceaser EK, Dickinson D, Zanoni G, et al. Cellular mechanisms of redox cell signalling: role of cysteine modification in controlling antioxidant defences in response to electrophilic lipid oxidation products. Biochem J 2004; (1) 378 (Pt 2): 373-82.

46. Kayanoki Y, Fujii J, Suzuki K, Kawata S, Matsuzawa Y, Taniguchi N. Suppression of antioxidative enzyme expression by transforming growth factor-beta 1 in rat hepatocytes. J Biol Chem 1994; 269 (22): 15488-92.

47. Arsalane K, Dubois CM, Muanza T, Bégin R, Boudreau F, Asselin C, et al. Transforming growth factor-betal is a potent inhibitor of glutathione synthesis in the lung epithelial cell line A549: transcriptional effect on the
GSH rate-limiting enzyme gamma-glutamylcysteine synthetase. Am J Respir Cell Mol Biol 1997; 17 (5): 599607.

48. Bakin AV, Stourman NV, Sekhar KR, Rinehart C, Yan $\mathrm{X}$, Meredith MJ, et al. Smad3-ATF3 signaling mediates TGF-beta suppression of genes encoding Phase II detoxifying proteins. Free Radic Biol Med 2005; 38 (3): 375-87.

49. Choi HK, Pokharel YR, Lim SC, Han HK, Ryu CS, Kim SK, et al. Inhibition of liver fibrosis by solubilized coenzyme Q10: Role of Nrf2 activation in inhibiting transforming growth factor-betal expression. Toxicol Appl Pharmacol 2009; 240 (3): 377-84.

50. Kawada N, Seki S, Kuroki T, Inoue M. Regulation of stellate cell proliferation by lipopolysaccharide: role of endogenous nitric oxide. J Gastroenterol Hepatol 1998; 13 Suppl: S6-13.

51. Kim KM, Yoon JH, Gwak GY, Kim W, Lee SH, Jang JJ, et al. Bile acid-mediated induction of cyclooxygenase-2 and Mcl-1 in hepatic stellate cells. Biochem Biophys Res Commun 2006; 342 (4): 1108-13.

52. Chen YX, Lu CH, Xie WF, Zhang XR, Zhang ZB, Wei $\mathrm{LX}$, et al. Effects of ribozyme targeting platelet-derived growth factor receptor beta subunit gene on the proliferation and apoptosis of hepatic stellate cells in vitro. Chin Med J (Engl) 2005; 118 (12): 982-8. 